\title{
АСИМПТОТИЧЕСКОЕ ПОВЕДЕНИЕ РЕШЕНИЙ ОБЫКНОВЕННЫХ ДИФФЕРЕНЦИАЛЬНЫХ УРАВНЕНИЙ $n$-ГО ПОРЯДКА С ПРАВИЛЬНО МЕНЯЮЩИМИСЯ НЕЛИНЕЙНОСТЯМИ
}

\author{
В. М. Евтухов, А. Г. Дорошенко \\ Одес. нац. ун-т им. И. И. Мечникова \\ ул. Дворянская, 2, Одесса, 65026, Украина
}

We establish conditions for the existence of some classes of solutions of the nonautonomous differential equations of the $n$th order with regularly varying nonlinearities and asymptotic representations of these solutions and their derivatives up to order $n-1$ inclusively as $t \uparrow \omega(\omega \leq+\infty)$.

Встановлюються умови існування деяких класів розв'язків неавтономного диференціального рівняння $n$-го порядку з правильно змінними нелінійностями, а також асимптотичні при $t \uparrow \omega$ $(\omega \leq+\infty)$ зображення для таких розв'язків та їх похідних до порядку $n-1$ включно.

1. Введение. Рассматривается уравнение

$$
y^{(n)}=\sum_{k=1}^{m} \alpha_{k} p_{k}(t) \prod_{j=0}^{n-1} \varphi_{k j}\left(y^{(j)}\right),
$$

где $\alpha_{k} \in\{-1 ; 1\}, k=\overline{1, m}, p_{k}:[a, \omega[\rightarrow] 0,+\infty[, k=\overline{1, m},-$ непрерывные функции, $\left.\varphi_{k j}: \triangle Y_{j} \rightarrow\right] 0,+\infty[, k=\overline{1, m}, j=\overline{0, n-1},-$ непрерывные и правильно меняющиеся при $y^{(j)} \rightarrow Y_{j}$ функции порядков $\sigma_{k j},-\infty<a<\omega \leq+\infty^{*}, \triangle Y_{j}-$ односторонняя окрестность $Y_{j}, Y_{j}$ равно либо 0, либо $\pm \infty$.

Определение 1.1. Непрерывная функция $\left.\varphi: \Delta_{Y} \rightarrow\right] 0,+\infty[$, где $Y$ равно либо нулю, либо $\pm \infty$ и $\Delta_{Y}-$ односторонняя окрестность $Y$, называется правильно меняющейся при $y \rightarrow Y$, если существует число $\sigma \in \mathbb{R}$ такое, что

$$
\lim _{\substack{y \rightarrow Y \\ y \in \Delta Y}} \frac{\varphi(\lambda y)}{\varphi(y)}=\lambda^{\sigma} \quad \text { для любого } \quad \lambda>0 .
$$

При этом число б называется порядком функции $\varphi$.

Частным случаем уравнения (1.1) является уравнение

$$
y^{(n)}=\alpha_{0} p(t) \varphi(y),
$$

где $\alpha_{0} \in\{-1,1\}, p:\left[a, \omega[\rightarrow] 0,+\infty\left[-\right.\right.$ непрерывная функция, а $\left.\varphi: \Delta_{Y_{0}} \longrightarrow\right] 0,+\infty[-$ непрерывная и правильно меняющаяся при $y \longrightarrow Y_{0}$ функция порядка $\sigma, Y_{0}$ равно либо нулю, либо $\pm \infty, \Delta_{Y_{0}}$ - односторонняя окрестность $Y_{0}$. Для такого уравнения в работе В. М. Евтухова и А. М. Самойленко [1] изучалась асимптотика так называемых $P_{\omega}\left(Y_{0}, \lambda_{0}\right)$ решений. Результаты данного исследования в работах В. М. Евтухова и А. М. Клопота [2 -4] частично распространены на случай уравнения вида (1.1).

${ }^{*}$ Считаем, что $a>1$ при $\omega=+\infty$ и $\omega-1<a<\omega$ при $\omega<+\infty$.

(C) В. М. Евтухов, А. Г. Дорошенко, 2018

ISSN 1562-3076. Нелінійні коливання, 2018, m. 21, № 2 
Определение 1.2. Решение уравнения (1.1) будем называть $P_{\omega}\left(Y_{0}, \ldots, Y_{n-1}, \lambda_{0}\right)$-решением, где $-\infty \leq \lambda_{0} \leq+\infty$, если оно определено на промежсутке $\left[t_{0}, \omega[\subset[a, \omega[\right.$ и удовлетворяет условиям

$$
\begin{gathered}
y^{(j)}(t) \in \Delta_{Y_{j}} \quad \text { npu } \quad t \in\left[t_{0}, \omega\left[, \quad \lim _{t \uparrow \omega} y^{(j)}(t)=Y_{j}, \quad j=\overline{0, n-1}\right.\right. \\
\lim _{t \uparrow \omega} \frac{\left[y^{(n-1)}(t)\right]^{2}}{y^{(n)}(t) y^{(n-2)}(t)}=\lambda_{0} .
\end{gathered}
$$

В [2 - 4] исследовалась ситуация, когда правая часть уравнения (1.1) на каждом из возможных типов $P_{\omega}\left(Y_{0}, \ldots, Y_{n-1}, \lambda_{0}\right)$-решений эквивалентна одному слагаемому, т. е. когда для некоторого $s \in\{1, \ldots, m\}$ справедливо

$$
\lim _{t \uparrow \omega} \frac{p_{k}(t) \prod_{j=0}^{n-1} \varphi_{k j}\left(y^{(j)}(t)\right)}{p_{s}(t) \prod_{j=0}^{n-1} \varphi_{s j}\left(y^{(j)}(t)\right)}=0 \quad \text { при } \quad k \in\{1, \ldots, m\} \backslash\{s\} .
$$

Целью настоящей работы является распространение некоторых из этих результатов на случай, когда на изучаемом классе решений в правой части такого уравнения имеется несколько главных слагаемых, т. е. когда для некоторого $s \in\{1, \ldots, m\}$ и не пустого множества $\Gamma \subset\{1, \ldots, m\}$

$$
\begin{gathered}
\lim _{t \uparrow \omega} \frac{p_{k}(t) \prod_{j=0}^{n-1} \varphi_{k j}\left(y^{(j)}(t)\right)}{p_{s}(t) \prod_{j=0}^{n-1} \varphi_{s j}\left(y^{(j)}(t)\right)}=0 \quad \text { при } \quad k \in\{1, \ldots, m\} \backslash \Gamma, \\
\lim _{t \uparrow \omega} \frac{p_{k}(t) \prod_{j=0}^{n-1} \varphi_{k j}\left(y^{(j)}(t)\right)}{p_{s}(t) \prod_{j=0}^{n-1} \varphi_{s j}\left(y^{(j)}(t)\right)}=c_{k s}=\mathrm{const} \neq 0 \quad \text { при } \mathrm{k} \in \Gamma .
\end{gathered}
$$

При этом будут исследованы $P_{\omega}\left(Y_{0}, \ldots, Y_{n-1}, \lambda_{0}\right)$-решения, для которых

$$
\lambda_{0} \in \mathbb{R} \backslash\left\{0, \frac{1}{2}, \frac{2}{3}, \ldots, \frac{n-2}{n-1}, 1\right\}
$$

и $\lambda_{0}=1$.

Выберем числа $b_{j} \in \Delta_{Y_{j}}, j=\overline{0, n-1}$, такими, чтобы выполнялись неравенства

$$
\left|b_{j}\right|<1 \quad \text { при } \quad Y_{j}=0, \quad b_{j}>1\left(b_{j}<-1\right) \quad \text { при } \quad Y_{j}=+\infty\left(Y_{j}=-\infty\right)
$$

и в дальнейшем, не ограничивая общности, будем считать, что

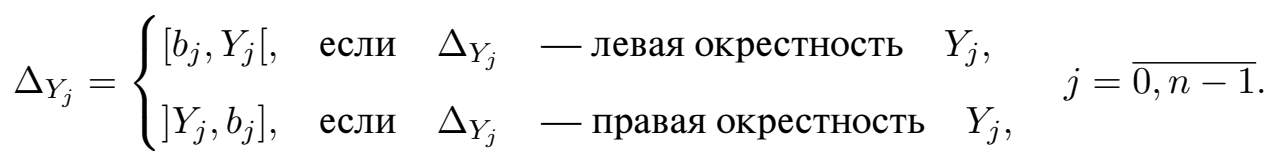

ISSN 1562-3076. Нелінійні коливання, 2018, m. 21, № 2 
Из определения $P_{\omega}\left(Y_{0}, \ldots, Y_{n-1}, \lambda_{0}\right)$-решения уравнения (1.1) и выбора $b_{j}, j=\overline{0, n-1}$, ясно, что каждое такое решение и все его производные до порядка $n-1$ включительно отличны от нуля на промежутке $\left[t_{0}, \omega[\right.$, причем на этом промежутке $(j+1)$-я производная данного решения положительна $(j \in\{0, \ldots, n-1\})$, если $\Delta_{Y_{j}}-$ левая окрестность $Y_{j}$, и отрицательна в противном случае. Учитывая этот факт, введем числа

$$
\begin{aligned}
& \nu_{0 j}=\operatorname{sign} b_{j},
\end{aligned}
$$

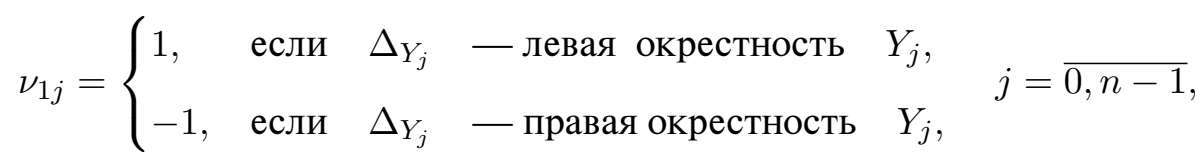

определяющие соответственно знаки $j$-й и $(j+1)$-й производных $P_{\omega}\left(Y_{0}, \ldots, Y_{n-1}, \lambda_{0}\right)$ решения. При этом заметим, что для $P_{\omega}\left(Y_{0}, \ldots, Y_{n-1}, \lambda_{0}\right)$-решения уравнения (1.1) выполняются условия

$$
\nu_{0 j} \nu_{1 j}<0, \quad \text { если } Y_{j}=0, \quad \nu_{0 j} \nu_{1 j}>0, \quad \text { если } \quad Y_{j}= \pm \infty \quad j=\overline{0, n-2} .
$$

2. Вспомогательные утверждения. В работе потребуются некоторые известные факты (см. [5]) из теории правильно меняющихся функций.

Определение 2.1. Правильно меняющаяся при $y \rightarrow Y$ функция $\left.L: \Delta_{Y} \longrightarrow\right] 0,+\infty[$, где $Y$ равно либо 0, либо $\pm \infty$ и $\Delta_{Y}-$ односторонняя окрестность $Y$, порядка $\sigma=0$ называется медленно меняющейся функцией.

В силу определений 1.1 и 2.1 правильно меняющаяся при $y \rightarrow Y$ функция $\varphi$ порядка $\sigma$ представима в виде

$$
\varphi(y)=|y|^{\sigma} L(y)
$$

где $L(y)$ - медленно меняющаяся функция при $y \rightarrow Y$.

Из результатов, представленных в монографии [5, с. 9-24] (гл. 1, пп. 1, 3, 5), вытекает следующее утверждение.

Лемма 2.1. Пусть $\left.L: \Delta_{Y} \longrightarrow\right] 0,+\infty[$ - медленно меняющаяся функция при $y \rightarrow Y$, где $Y$ равно либо 0, либо $\pm \infty$. Тогда:

1) для любого $\lambda>0$

$$
\lim _{\substack{y \rightarrow Y \\ y \in \Delta Y}} \frac{L(\lambda y)}{L(y)}=1
$$

$u$ это предельное соотношение выполняется равномерно по $\lambda$ на любом промежутке $[c, d] \subset$ $\subset] 0,+\infty[$;

2) существует непрерывно дифференцируемая медленно меняющаяся при $y \rightarrow Y$ функция $\left.L_{0}: \Delta_{Y} \longrightarrow\right] 0,+\infty[$ такая, что

$$
\lim _{\substack{y \rightarrow Y \\ y \in \Delta_{Y}}} \frac{L(y)}{L_{0}(y)}=1 \quad u \quad \lim _{\substack{y \rightarrow Y \\ y \in \Delta_{Y}}} \frac{y L_{0}^{\prime}(y)}{L_{0}(y)}=0 ;
$$

3) справедливо предельное соотношение

$$
\lim _{\substack{y \rightarrow Y \\ y \in \Delta_{y}}} \frac{\ln L(y)}{\ln |y|}=0
$$


Определение 2.2 [4, с. 54]. Будем говорить, что медленно меняющаяся при $y \rightarrow Y$ функция $\left.L: \Delta_{Y} \rightarrow\right] 0,+\infty\left[\left(Y\right.\right.$ равно либо 0, либо $\pm \infty, \Delta_{Y}-$ односторонняя окрестность $\left.Y\right)$ удовлетворяет условию $S$, если

$$
L\left(\nu e^{[1+o(1)] \ln |y|}\right)=L(y)[1+o(1)] \quad \text { npu } \quad y \rightarrow Y \quad\left(y \in \Delta_{Y}\right),
$$

где $\nu=\operatorname{sign} y$.

Замечание 2.1 [1, с. 56]. Если функция $\left.L: \Delta_{Y} \longrightarrow\right] 0,+\infty[$ удовлетворяет условию $S$, а функция $y:\left[t_{0}, \omega\left[\longrightarrow \Delta_{Y}\right.\right.$ непрерывно дифференцируема и такова, что

$$
\lim _{t \uparrow \omega} y(t)=Y, \quad \frac{y^{\prime}(t)}{y(t)}=\frac{\xi^{\prime}(t)}{\xi(t)}[r+o(1)] \quad \text { при } \quad t \uparrow \omega,
$$

где $r$ - отличная от нуля вещественная постоянная, $\xi-$ непрерывно дифференцируемая в некоторой левой окрестности $\omega$ вещественная функция, для которой $\xi^{\prime}(t) \neq 0$, то

$$
L(y(t))=L\left(\nu_{0}|\xi(t)|^{r}\right)[1+o(1)] \quad \text { при } \quad t \uparrow \omega \quad\left(\nu_{0}=\operatorname{sign} y(t)\right) .
$$

Для того чтобы описать априорные свойства исследуемых типов $P_{\omega}\left(Y_{0}, \ldots, Y_{n-1}, \lambda_{0}\right)$ решений, положим

$$
\begin{gathered}
a_{0 i}=(n-i) \lambda_{0}-(n-i-1), \quad i=1, \ldots, n, \quad \text { при } \lambda_{0} \in \mathbb{R}, \\
\pi_{\omega}(t)=\left\{\begin{array}{lll}
t, & \text { если } \omega=+\infty, \\
t-\omega, & \text { если } \omega<+\infty .
\end{array}\right.
\end{gathered}
$$

Лемма 2.2 [1]. Пусть $y:\left[t_{0}, \omega\left[\longrightarrow \Delta_{Y_{0}}-\right.\right.$ произвольное $P_{\omega}\left(Y_{0}, \ldots, Y_{n-1}, \lambda_{0}\right)$-решение уравнения (1.1). Тогда:

1) если $\lambda_{0} \in \mathbb{R} \backslash\left\{0, \frac{1}{2}, \frac{2}{3}, \ldots, \frac{n-2}{n-1}, 1\right\}$, то при $t \uparrow \omega$ имеют место асимптотические соотношения

$$
y^{(j)}(t) \sim \frac{\left[\left(\lambda_{0}-1\right) \pi_{\omega}(t)\right]^{n-j-1}}{\prod_{i=j+1}^{n-1} a_{0 i}} y^{(n-1)}(t), \quad j=0,1, \ldots, n-2, \quad y^{(n)}(t) \sim \frac{y^{(n-1)}(t)}{\left(\lambda_{0}-1\right) \pi_{\omega}(t)} ;
$$

2) если $\lambda_{0}=1$, то

$$
\frac{y^{\prime}(t)}{y(t)} \sim \frac{y^{\prime \prime}(t)}{y^{\prime}(t)} \sim \cdots \sim \frac{y^{(n)}(t)}{y^{(n-1)}(t)} \quad n p u \quad t \uparrow \omega \quad u \quad \lim _{t \uparrow \omega} \frac{\pi_{\omega}(t) y^{\prime}(t)}{y(t)}= \pm \infty .
$$

Наряду с понятиями и свойствами правильно меняющихся функций, в работе будут также использоваться некоторые известные результаты о существовании исчезающих решений системы квазилинейных дифференциальных уравнений вида

$$
\frac{d v_{i}}{d t}=h(t)\left[f_{i}\left(t, v_{1}, \ldots, v_{n}\right)+\sum_{j=1}^{n} a_{i j} v_{j}+V_{i}\left(t, v_{1}, \ldots, v_{n}\right)\right], \quad i=\overline{1, n}
$$

ISSN 1562-3076. Нелінійні коливання, 2018, m. 21, № 2 
в которой $a_{i j} \in R, i, j=\overline{1, n}, h:\left[t_{0}, \omega\left[\rightarrow R, i, j=\overline{1, n},-\right.\right.$ непрерывная функция и $f_{i}$, $V_{i}:\left[t_{0}, \omega\left[\times R_{c}^{n} \rightarrow R, i=\overline{1, n},-\right.\right.$ непрерывные функции, удовлетворяющие условиям

$$
\begin{gathered}
\lim _{t \uparrow \omega} f_{i}\left(t, v_{1}, \ldots, v_{n}\right)=0, \quad i=\overline{1, n}, \quad \text { равномерно по } \quad\left(v_{1}, \ldots, v_{n}\right) \in R_{c}^{n}, \\
\lim _{\left|v_{1}\right|+\cdots+\left|v_{n}\right| \rightarrow 0} \frac{V_{i}\left(t, v_{1}, \ldots, v_{n}\right)}{\left|v_{1}\right|+\cdots+\left|v_{n}\right|}=0, \quad i=\overline{1, n}, \quad \text { равномерно по } \quad t \in\left[t_{0}, \omega[,\right.
\end{gathered}
$$

где

$$
-\infty<t_{0}<\omega \leq+\infty, \quad R_{c}^{n}=\left\{\left(v_{1}, \ldots, v_{n}\right) \in R^{n}:\left|v_{i}\right| \leq c, i=1, \ldots, n\right\}, \quad c>0 .
$$

В работе В. М. Евтухова и А. М. Самойленко [6] установлен следующий результат.

Лемма 2.3. Пусть $h:\left[t_{0}, \omega[\rightarrow R-\right.$ непрерывная функция такая, что

$$
h(t) \neq 0 \quad \text { npu } \quad t \in\left[t_{0}, \omega\left[, \quad \int_{t_{0}}^{\omega} h(t) d t= \pm \infty .\right.\right.
$$

Пусть, кроме того, матрииа $A=\left(a_{i j}\right)_{i, j=1}^{n}$ не имеет собственных значений с нулевой действительной частью. Тогда система дифференциальных уравнений (2.7) имеет хотя бы одно решение $\left(v_{i}\right)_{i=1}^{n}:\left[t_{1},+\infty\left[\rightarrow R_{c}^{n}\left(t_{1} \in\left[t_{0}, \omega[)\right.\right.\right.\right.$, стремящееся $\kappa$ нулю при $t \uparrow \omega$, причем таких решений существует m-параметрическое семейство, если среди собственных значений матрицы А имеется $m$ собственных значений (с учетом кратных), действительные части которых имеют знак, противоположный знаку функции $h$ на промежутке $\left[t_{0}, \omega[\right.$.

3. Основные результаты. Для формулировки первого основного результата, относящегося к случаю $\lambda_{0} \in \mathbb{R} \backslash\left\{0, \frac{1}{2}, \frac{2}{3}, \ldots, \frac{n-2}{n-1}, 1\right\}$, учитывая (2.3) и (2.4), введем следующие обозначения для $k=\overline{1, m}$ :

$$
\begin{gathered}
\gamma_{k}=1-\sum_{j=0}^{n-1} \sigma_{k j}, \quad \mu_{k n}=\sum_{j=0}^{n-2} \sigma_{k j}(n-j-1), \\
C_{k}=\prod_{j=0}^{n-2}\left|\frac{\left(\lambda_{0}-1\right)^{n-j-1}}{\prod_{i=j+1}^{n-1} a_{0 i}}\right|^{\sigma_{k j}}, \quad J_{k n}(t)=\int_{A_{k n}}^{t} p_{k}(\tau)\left|\pi_{\omega}(\tau)\right|^{\mu_{k n}} d \tau,
\end{gathered}
$$

где

$$
A_{k n}=\left\{\begin{array}{lll}
a, & \text { если } & \int_{a}^{\omega} p_{k}(t)\left|\pi_{\omega}(t)\right|^{\mu_{k n}} d t=+\infty, \\
\omega, & \text { если } & \int_{a}^{\omega} p_{k}(t)\left|\pi_{\omega}(t)\right|^{\mu_{k n}} d t<+\infty .
\end{array}\right.
$$

Теорема 3.1. Пусть $\lambda_{0} \in \mathbb{R} \backslash\left\{0, \frac{1}{2}, \ldots, \frac{n-2}{n-1}, 1\right\}$. Пусть, кроме того, для некоторого $s \in\{1, \ldots, m\}$ выполняется неравенство $\gamma_{s} \neq 0$ и для непустого множества $\Gamma \subset\{1, \ldots, m\}$ 
медленно меняющиеся составляющие $L_{k j}(y)$ функций $\varphi_{k j}$ удовлетворяют условию $S$ для каждого $k \in \Gamma$ и $j \in\{0, \ldots, n-1\}$. Тогда для существования $P_{\omega}\left(Y_{0}, \ldots, Y_{n-1}, \lambda_{0}\right)$-решения уравнения (1.1), для которого выполняется (1.3), (1.4) и $\sum_{k \in \Gamma} \alpha_{k} c_{k s} \neq 0$, необходимо, чтобы наряду с (1.6) выполнялись неравенства

$$
\begin{gathered}
\nu_{0 j} \nu_{1 j} a_{0 j+1}\left(\lambda_{0}-1\right) \pi_{\omega}(t)>0, \quad j=\overline{0, n-2}, \\
\left.\nu_{0 n-1} \gamma_{s}\left(\sum_{k \in \Gamma} \alpha_{k} c_{k s}\right) J_{s n}(t)>0 \quad n p u \quad t \in\right] a, \omega[,
\end{gathered}
$$

а также условия

$$
\begin{gathered}
\lim _{t \uparrow \omega} \frac{\pi_{\omega}(t) J_{s n}^{\prime}(t)}{J_{s n}(t)}=\frac{\gamma_{s}}{\lambda_{0}-1}, \\
\lim _{t \uparrow \omega} \frac{p_{k}(t) \prod_{j=0}^{n-1} \varphi_{k j}\left(Y_{j}(t)\right)}{p_{s}(t) \prod_{j=0}^{n-1} \varphi_{s j}\left(Y_{j}(t)\right)}=0 \quad n p u \quad k \in\{1, \ldots, m\} \backslash\{s\}, \\
\lim _{t \uparrow \omega} \frac{p_{k}(t) \prod_{j=0}^{n-1} \varphi_{k j}\left(Y_{j}(t)\right)}{p_{s}(t) \prod_{j=0}^{n-1} \varphi_{s j}\left(Y_{j}(t)\right)}=c_{k s} \quad \text { npu } \quad k \in \Gamma,
\end{gathered}
$$

где

$$
\begin{gathered}
Y_{j}(t)=\nu_{0 n-1} \frac{\left[\left(\lambda_{0}-1\right) \pi_{\omega}(t)\right]^{n-j-1}}{\prod_{k=j+1}^{n-1} a_{0 k}}|Y(t)|^{1 / \gamma_{s}}, \\
Y(t)=\gamma_{s} C_{s}\left(\sum_{k \in \Gamma} \alpha_{k} c_{k s}\right) J_{s n}(t) \prod_{j=0}^{n-1} L_{s j}\left(\nu_{0 j}\left|\pi_{\omega}(t)\right|^{\frac{a_{0 j+1}}{\lambda_{0}-1}}\right) .
\end{gathered}
$$

Более того, для каждого такого решения при $t \uparrow \omega$ имеют место асимптотические представления

$$
y^{(j-1)}(t)=Y_{j-1}(t)[1+o(1)], \quad j=\overline{1, \ldots, n} .
$$

Доказательство. Пусть $y:\left[t_{0}, \omega\left[\longrightarrow \Delta_{Y_{0}}\right.\right.$ является $P_{\omega}\left(Y_{0}, \ldots, Y_{n-1}, \lambda_{0}\right)$-решением уравнения (1.1), для которого выполняются условия (1.3), (1.4) и $\sum_{k \in \Gamma} \alpha_{k} c_{k s} \neq 0$. Тогда, как было указано ранее, справедливы неравенства (1.6) и в силу леммы 2.2 имеют место асимптотические соотношения (2.5), из которых следует, что

$$
\frac{y^{(j+1)}(t)}{y^{(j)}(t)}=\frac{a_{0 j+1}}{\left(\lambda_{0}-1\right) \pi_{\omega}(t)}[1+o(1)], \quad j=\overline{0, n-1}, \quad \text { при } \quad t \uparrow \omega,
$$

откуда с учетом (1.5) выполняются первые из неравенств (3.1). 
Учитывая условия (1.3), (1.4) из (1.1), получаем

$$
y^{(n)}(t)=\left(\sum_{k \in \Gamma} \alpha_{k} c_{k s}\right) p_{s}(t) \prod_{j=0}^{n-1} \varphi_{s j}\left(y^{(j)}(t)\right)[1+o(1)] \quad \text { при } \quad t \uparrow \omega .
$$

Отсюда с учетом представления правильно меняющихся функций через медленно меняющиеся и асимптотических соотношений (2.5) имеем

$$
\frac{y^{(n)}(t)\left|y^{(n-1)}(t)\right|^{\gamma_{s}-1}}{\prod_{j=0}^{n-1} L_{s j}\left(y^{(j)}(t)\right)}=C_{s}\left(\sum_{k \in \Gamma} \alpha_{k} c_{k s}\right) p_{s}(t)\left|\pi_{\omega}(t)\right|^{\mu_{s n}}[1+o(1)] \quad \text { при } \quad t \uparrow \omega .
$$

Интегрируя это соотношение на промежутке от $t_{0}$ до $t$, если $A_{s n}=a$, и на промежутке от $t$ до $\omega$, если $A_{s n}=\omega$, получаем

$$
\int_{A_{s n}}^{t} \frac{y^{(n)}(\tau)\left|y^{(n-1)}(\tau)\right|^{\gamma_{s}-1}}{\prod_{j=0}^{n-1} L_{s j}\left(y^{(j)}(\tau)\right)} d \tau=C_{s}\left(\sum_{k \in \Gamma} \alpha_{k} c_{k s}\right) J_{s n}(t)[1+o(1)] \quad \text { при } \quad t \uparrow \omega .
$$

В силу леммы 2.1 существуют непрерывно дифференцируемые функции $L_{0 k j}: \Delta_{Y_{j}} \rightarrow$ $\rightarrow] 0,+\infty[$ такие, что при $k=\overline{1, m}, j=\overline{0, n-1}$

$$
L_{k j}\left(y^{(j)}\right) \sim L_{0 k j}\left(y^{(j)}\right) \quad \text { при } \quad y^{(j)} \rightarrow Y_{j}, \quad \lim _{y^{(j)} \rightarrow Y_{j}} \frac{y^{(j)} L_{0 k j}^{\prime}\left(y^{(j)}\right)}{L_{0 k j}\left(y^{(j)}\right)}=0 .
$$

Учитывая условия леммы 2.1, с использованием правила Лопиталя находим

$$
\begin{aligned}
& \lim _{t \uparrow \omega} \frac{\frac{\left|y^{(n-1)}(t)\right|^{\gamma_{s}}}{\prod_{j=0}^{n-1} L_{0 s j}\left(y^{(j)}(t)\right)}}{\int_{B_{s n}}^{t} \frac{y^{(n)}(\tau)\left|y^{(n-1)}(\tau)\right|^{\gamma_{s}-1}}{\prod_{j=0}^{n-1} L_{s j}\left(y^{(j)}(\tau)\right)} d \tau}= \\
& =\operatorname{sign} y^{(n-1)}(t) \lim _{t \uparrow \omega} \frac{\prod_{j=0}^{n-1} L_{s j}\left(y^{(j)}(t)\right)}{\prod_{j=0}^{n-1} L_{0 s j}\left(y^{(j)}(t)\right)} \times \\
& \times\left[\gamma_{s}-\frac{y^{(n-1)}(t)}{y^{(n)}(t)} \sum_{j=0}^{n-1} \frac{y^{(j)}(t) L_{o s j}^{\prime}\left(y^{(j)}(t)\right)}{L_{o s j}\left(y^{(j)}(t)\right)} \frac{y^{(j+1)}(t)}{y^{(j)}(t)}\right]= \\
& =\nu_{0 n-1} \gamma_{s} .
\end{aligned}
$$

В силу этого предельного соотношения из (3.7) с учетом (3.8) следует, что

$$
\frac{\left|y^{(n-1)}(t)\right|^{\gamma_{s}}}{\prod_{j=0}^{n-1} L_{s j}\left(y^{(j)}(t)\right)}=\nu_{0 n-1} \gamma_{s} C_{s}\left(\sum_{k \in \Gamma} \alpha_{k} c_{k s}\right) J_{s n}(t)[1+o(1)] \quad \text { при } \quad t \uparrow \omega,
$$

откуда, в частности, вытекает выполнение второго из неравенств (3.1). 
Так как функции $L_{s j}$ удовлетворяют условию $S$, то с учетом замечания 2.1 выполнено равенство

$$
L_{s j}\left(y_{j}(t)\right)=L_{s j}\left(\nu_{0 j}\left|\pi_{\omega}(t)\right|^{\frac{a_{0 j+1}}{\lambda_{0}-1}}\right)[1+o(1)] \quad \text { при } \quad t \uparrow \omega, \quad j=\overline{0, n-1},
$$

поэтому из (3.9) получим

$$
y^{(n-1)}(t)=\nu_{0 n-1}\left|\prod_{j=0}^{n-1} L_{s j}\left(\nu_{j}\left|\pi_{\omega}(t)\right|^{\frac{a_{0 j+1}}{\lambda_{0}-1}}\right) \gamma_{s} C_{s} J_{s n}(t)\left(\sum_{k \in \Gamma} \alpha_{k} c_{k s}\right)\right|^{1 / \gamma_{s}}[1+o(1)] \quad \text { при } \quad t \uparrow \omega .
$$

Отсюда вытекают представления (3.5), и из (3.9), (3.6) находим, что

$$
\frac{y^{(n)}(t)}{y^{(n-1)}(t)}=\frac{p_{s}(t)\left|\pi_{\omega}(t)\right|^{\mu_{s n}}}{\gamma_{s} J_{s n}(t)}[1+o(1)] \quad \text { при } \quad t \uparrow \omega .
$$

Поэтому в силу последнего из предельных соотношений (2.5) выполняется условие (3.2).

Теорема 3.1 доказана.

Теорема 3.2. Пусть выполнены условия теоремы 3.1 и наряду с (1.6), (3.2)-(3.4) алгебраическое относительно о уравнение

$$
\sum_{m=0}^{n-1} B_{m} \prod_{i=m+1}^{n-1} a_{0 i} \prod_{j=1}^{m}\left(a_{0 j}+\rho\right)=\prod_{j=1}^{n}\left(a_{0 j}+\rho\right)
$$

где

$$
B_{m}=\frac{\sum_{k \in \Gamma} \alpha_{k} c_{k s} \sigma_{k m}}{\sum_{k \in \Gamma} \alpha_{k} c_{k s}}
$$

не имеет корней с нулевой действительной частью. Тогда дифференциальное уравнение (1.1) имеет $P_{\omega}\left(Y_{0}, \ldots, Y_{n-1}, \lambda_{0}\right)$-решение вида (3.5), причем существует l-параметрическое семейство решений с такими представлениями в случае, когда среди корней алгебрачческого уравнения (3.10) имеется $l$ (с учетом кратных) корней, действительные части которых имеют знак, противоположный знаку функции $\pi_{\omega}(t)\left(\lambda_{0}-1\right)$.

Доказательство. Применим к уравнению (1.1) преобразование

$$
y^{(i-1)}(t)=\nu_{0 n-1}\left|Y_{0}(t)\right|^{1 / \gamma_{s}} \frac{\left[\left(\lambda_{0}-1\right) \pi_{\omega}(t)\right]^{n-i}}{\prod_{k=i}^{n-1} a_{0 k}}\left[1+v_{i}(\tau)\right], \quad i=\overline{1, n}, \quad \tau=\beta \ln \left|\pi_{\omega}(t)\right|,
$$

где

$$
\beta=\left\{\begin{array}{lll}
1, & \text { если } & \omega=+\infty \\
-1, & \text { если } & \omega<+\infty
\end{array}\right.
$$

И

$$
Y_{0}(t)=\gamma_{s} C_{s} J_{s n}(t) \prod_{j=0}^{n-1} L_{0 s j}\left(\nu_{0 j}\left|\pi_{\omega}(t)\right|^{\frac{a_{0 j+1}}{\lambda_{0}-1}}\right) \sum_{k \in \Gamma} \alpha_{k} c_{k s} .
$$

ISSN 1562-3076. Нелінійні коливання, 2018, m. 21, № 2 
В результате преобразования получим систему дифференциальных уравнений вида

$$
\left\{\begin{array}{l}
v_{i}^{\prime}=\frac{\beta}{\lambda_{0}-1}\left[a_{0 i}\left(1+v_{i+1}\right)-\left((n-i)\left(\lambda_{0}-1\right)+h(\tau)\right)\left(1+v_{i}\right)\right], \quad i=\overline{1, n-2} \\
v_{n-1}^{\prime}=\frac{\beta}{\lambda_{0}-1}\left[a_{0 n-1}\left(1+v_{n}\right)-\left(\left(\lambda_{0}-1\right)+h(\tau)\right)\left(1+v_{n-1}\right)\right] \\
v_{n}^{\prime}=\frac{\beta}{\lambda_{0}-1}\left[G\left(\tau, v_{1}, \ldots, v_{n}\right)-h(\tau)\left[1+v_{n}\right]\right]
\end{array}\right.
$$

в которой

$$
G\left(\tau(t), v_{1}, \ldots, v_{n}\right)=\frac{\nu_{0 n-1}\left(\lambda_{0}-1\right) \pi_{\omega}(t)}{\left|Y_{0}(t)\right|^{1 / \gamma_{s}}} \sum_{k=1}^{m} \alpha_{k} p_{k}(t) \prod_{j=0}^{n-1} \varphi_{k j}\left(Y_{j}^{0}(t)\left[1+v_{j+1}\right]\right),
$$

где

И

$$
Y_{j}^{0}(t)=\nu_{0 n-1} \frac{\left[\left(\lambda_{0}-1\right) \pi_{\omega}(t)\right]^{n-j-1}}{\prod_{k=j+1}^{n-1} a_{0 k}}\left|Y_{0}(t)\right|^{1 / \gamma_{s}}
$$

$$
h(\tau(t))=\frac{\left(\lambda_{0}-1\right) \pi_{\omega}(t) Y_{0}^{\prime}(t)}{\gamma_{s} Y_{0}(t)} .
$$

Правые части этой системы непрерывны на множестве $\left[\tau_{0},+\infty\left[\times \mathbb{R}_{1 / 2}^{n}\right.\right.$, где

$$
\left.\mathbb{R}_{1 / 2}^{n}=\left\{\left(v_{1}, \ldots, v_{n}\right) \in \mathbb{R}^{n} ;\left|v_{i}\right| \leq \frac{1}{2}, i=\overline{1, n}\right)\right\}, \quad \tau_{0}=\beta \ln \left|\pi_{\omega}(a)\right| .
$$

Кроме того, в силу условия (3.2) и (3.8)

$$
\lim _{\tau \rightarrow+\infty} h(\tau(t))=\lim _{t \uparrow \omega} h(t)=\lim _{t \uparrow \omega} \frac{\pi_{\omega}(t)\left(\lambda_{0}-1\right) Y_{0}^{\prime}(t)}{\gamma_{s} Y_{0}(t)}=1
$$

Далее, получим удобное для дальнейшего использования представление функции $G$. В силу представления правильно меняющейся функции через медленно меняющуюся, условия 1 леммы 2.1 и соотношения (3.12), получаем

$$
\begin{aligned}
\varphi_{k j}\left(Y_{j}^{0}(t)\left(1+v_{j+1}\right)\right) & =\left|Y_{j}^{0}(t)\left(1+v_{j+1}\right)\right|^{\sigma_{k j}} L_{k j}\left(Y_{j}^{0}(t)\left(1+v_{j+1}\right)\right)= \\
& =\left|1+v_{j+1}\right|^{\sigma_{k j}}\left[1+r_{k j}(t)\right] \varphi_{k j}\left(Y_{j}^{0}(t)\right),
\end{aligned}
$$

где $k=\overline{1, m}, j=\overline{0, n-1}$, функции $r_{k j}$ таковы, что $\lim _{t \uparrow \omega} r_{k j}\left(t, v_{j+1}\right)=0$ равномерно по $\left(v_{j+1}\right) \in \mathbb{R}_{1 / 2}^{2}$.

Учитывая соотношения (3.15), получаем

$$
\begin{aligned}
& \frac{\nu_{0 n-1} \pi_{\omega}(t)}{\left|Y_{0}(t)\right|^{1 / \gamma_{s}}} \sum_{k=1}^{m} \alpha_{k} p_{k}(t) \prod_{j=0}^{n-1} \varphi_{k j}\left(Y_{j}^{0}(t)\left[1+v_{j+1}\right]\right)= \\
& \quad=\frac{\nu_{0 n-1} \pi_{\omega}(t)}{\left|Y_{0}(t)\right|^{1 / \gamma_{s}}} \sum_{k=1}^{m} \alpha_{k} p_{k}(t) \prod_{j=0}^{n-1}\left|Y_{j}^{0}(t)\right|^{\sigma_{k j}}\left|1+v_{j+1}\right|^{\sigma_{k j}} L_{k j}\left(Y_{j}^{0}(t)\right)\left[1+r_{k j}\left(t, v_{j+1}\right)\right]=
\end{aligned}
$$


АСИМПТОТИЧЕСКОЕ ПОВЕДЕНИЕ РЕШЕНИЙ ОБЫКНОВЕННЫХ ДИФФЕРЕНЦИАЛЬНЫХ УРАВНЕНИЙ...

$$
=\frac{\nu_{0 n-1} \pi_{\omega}(t)}{\left|Y_{0}(t)\right|^{1 / \gamma_{s}}} \sum_{k=1}^{m} \alpha_{k} p_{k}(t)\left(\prod_{j=0}^{n-1} \varphi_{k j}\left(Y_{j}^{0}(t)\right)\left|1+v_{j+1}\right|^{\sigma_{k j}}\right)\left[1+r_{k}\left(t, v_{1}, \ldots, v_{n}\right)\right],
$$

где $\lim _{t \uparrow \omega} r_{k}\left(t, v_{1}, \ldots, v_{n}\right)=0$ равномерно по $\left(v_{1}, \ldots, v_{n}\right) \in \mathbb{R}_{1 / 2}^{n}$.

Таким образом, с учетом предельных соотношений (3.3) и (3.4) имеем

$$
\begin{aligned}
\frac{\nu_{0 n-1} \pi_{\omega}(t)}{\left|Y_{0}(t)\right|^{\frac{1}{\gamma_{s}}}} p_{s}(t) \prod_{j=0}^{n-1} \varphi_{s j}\left(Y_{j}^{0}(t)\right) \times & {\left[\sum_{k \in \Gamma} \frac{\left.\alpha_{k} p_{k}(t) \prod_{j=0}^{n-1} \varphi_{k j}\left(Y_{j}^{0}(t)\right)\left[1+r_{k}\left(1, v_{1}, \ldots, v_{n}\right)\right] \prod_{j=0}^{n-1}\left|1+v_{j+1}\right|^{\sigma_{k j}}\right]}{p_{s}(t) \prod_{j=0}^{n-1} \varphi_{s j}\left(Y_{j}^{0}(t)\right)}\right]+} \\
+ & \frac{\nu_{0 n-1} \pi_{\omega}(t)}{\left|Y_{0}(t)\right|^{\frac{1}{\gamma_{s}}}} p_{s}(t) \prod_{j=0}^{n-1} \varphi_{s j}\left(Y_{j}^{0}(t)\right) \times \\
\times & {\left[\sum_{k \in\{1, \ldots, m\} \backslash \Gamma} \frac{\alpha_{k} p_{k}(t) \prod_{j=0}^{n-1} \varphi_{k j}\left(Y_{j}^{0}(t)\right)\left[1+r_{k}\left(1, v_{1}, \ldots, v_{n}\right)\right] \prod_{j=0}^{n-1}\left|1+v_{j+1}\right|^{\sigma_{k j}}}{p_{s}(t) \prod_{j=0}^{n-1} \varphi_{s j}\left(Y_{j}^{0}(t)\right)}\right]=} \\
= & \frac{\nu_{0 n-1} \pi_{\omega}(t)}{\left|Y_{0}(t)\right|^{\frac{1}{\gamma_{s}}}} p_{s}(t) \prod_{j=0}^{n-1} \varphi_{s j}\left(Y_{j}^{0}(t)\right)\left[\sum_{k \in \Gamma} \alpha_{k} c_{k s} \prod_{j=0}^{n-1}\left|1+v_{j+1}\right|^{\sigma_{k j}}+R_{k}\left(t, v_{1}, \ldots, v_{n}\right)\right] .
\end{aligned}
$$

С учетом предельных соотношений (3.3) и (3.4) получаем

$$
\frac{\nu_{0 n-1} \pi_{\omega}(t)}{\left|Y_{0}(t)\right|^{1 / \gamma_{s}}} p_{s}(t) \prod_{j=0}^{n-1} \varphi_{s j}\left(Y_{j}^{0}(t)\right)\left[\sum_{k \in \Gamma} \alpha_{k} c_{k s} \prod_{j=0}^{n-1}\left|1+v_{j+1}\right|^{\sigma_{k j}}+R_{k}\left(t, v_{1}, \ldots, v_{n}\right)\right] \text {, }
$$

где $\lim _{t \uparrow \omega} R_{k}\left(t, v_{1}, \ldots, v_{n}\right)=0$, равномерно по $\left(v_{1}, \ldots, v_{n}\right) \in \mathbb{R}_{1 / 2}^{n}$. Здесь, в силу (3.8) множитель перед скобкой имеет вид

$$
\begin{gathered}
\frac{\nu_{0 n-1} \pi_{\omega}(t)}{\left|Y_{0}(t)\right|^{1 / \gamma_{s}}} p_{s}(t) \prod_{j=0}^{n-1} \varphi_{s j}\left(Y_{j}^{0}(t)\right)=\frac{\nu_{0 n-1} \pi_{\omega}(t)}{\left|Y_{0}(t)\right|^{1 / \gamma_{s}}} p_{s}(t) \prod_{j=0}^{n-1}\left|Y_{j}^{0}(t)\right|^{\sigma_{s j}} L_{0 s j}\left(Y_{j}^{0}(t)\right)= \\
=\frac{\nu_{0 n-1} \pi_{\omega}(t) p_{s}(t)\left|\pi_{\omega}(t)\right|^{\mu_{s n}} C_{s} \prod_{j=0}^{n-1} L_{0 s j}\left(Y_{j}^{0}(t)\right)}{\left|\gamma_{s} C_{s} J_{s n}(t) \prod_{j=0}^{n-1} L_{0 s j}\left(\nu_{0 j}\left|\pi_{\omega}(t)\right|^{\frac{a_{0 j+1}}{\lambda_{0}-1}}\right) \sum_{k \in \Gamma} \alpha_{k} c_{k s}\right|} \sim \\
\sim \frac{\nu_{0 n-1} \pi_{\omega}(t) p_{s}(t)\left|\pi_{\omega}(t)\right|^{\mu_{s n}} C_{s} \prod_{j=0}^{n-1} L_{s j}\left(Y_{j}^{0}(t)\right)}{\left|\gamma_{s} C_{s} J_{s n}(t) \prod_{j=0}^{n-1} L_{s j}\left(\nu_{0 j}\left|\pi_{\omega}(t)\right|^{\frac{a_{0 j+1}}{\lambda_{0}-1}}\right) \sum_{k \in \Gamma} \alpha_{k} c_{k s}\right|} \quad \text { при } t \rightarrow \omega .
\end{gathered}
$$

ISSN 1562-3076. Нелінійні коливання, 2018, m. 21, № 2 
Принимая во внимание эти соотношения, второе из неравенств (3.1) и то, что функции $L_{s j}$ удовлетворяют условию $S$, последнее уравнение системы дифференциальных уравнений запишем в виде

$$
\begin{aligned}
v_{n}^{\prime}= & -\frac{\beta}{\lambda_{0}-1}(1+\xi(\tau))\left(1+v_{n}\right)+\frac{\beta}{\lambda_{0}-1} \frac{1}{\sum_{k \in \Gamma} \alpha_{k} c_{k s}} \times \\
& \times\left(\sum_{k \in \Gamma} \alpha_{k} c_{k s} \prod_{j=0}^{n-1}\left|1+v_{j+1}\right|^{\sigma_{k j}}\left(1+\widetilde{R}_{k}\left(\tau, v_{1}, \ldots, v_{n}\right)\right)+\widetilde{\widetilde{R}}_{k}\left(\tau, v_{1}, \ldots, v_{n}\right)\right) .
\end{aligned}
$$

Исходя из предыдущих преобразований, в силу (3.14) система дифференциальных уравнений (3.13) принимает следующий вид:

$$
\left\{\begin{array}{l}
v_{i}^{\prime}=\frac{\beta}{\lambda_{0}-1}\left[f_{i}\left(\tau, v_{1}, \ldots, v_{n}\right)+a_{0 i}\left(1+v_{i+1}\right)-a_{0 i}\left(1+v_{i}\right)\right], \quad i=\overline{1, n-2}, \\
v_{n-1}^{\prime}=\frac{\beta}{\lambda_{0}-1}\left[f_{n-1}\left(\tau, v_{1}, \ldots, v_{n}\right)+a_{0 n-1}\left(1+v_{n}\right)-a_{0 n-1}\left(1+v_{n-1}\right)\right], \\
v_{n}^{\prime}=\frac{\beta}{\lambda_{0}-1}\left[f_{n}\left(\tau, v_{1}, \ldots, v_{n}\right)-\left(1+v_{n}\right)+\frac{1}{\sum_{k \in \Gamma} \alpha_{k} c_{k s}} \sum_{k \in \Gamma} \alpha_{k} c_{k s} \prod_{j=0}^{n-1}\left|1+v_{j+1}\right|^{\sigma_{k j}}\right],
\end{array}\right.
$$

где функции $f_{i}, i=\overline{1, n}$, непрерывны на множестве $\left[\tau_{0},+\infty\left[\times \mathbb{R}_{1 / 2}^{n}\right.\right.$ и таковы, что

$$
\lim _{\tau \rightarrow+\infty} f_{i}\left(\tau, v_{1}, \ldots, v_{n}\right)=0 \quad \text { равномерно по } \quad\left(v_{1}, \ldots, v_{n}\right) \in \mathbb{R}_{1 / 2}^{n} .
$$

Выделим в последнем слагаемом системы (3.13) линейную часть:

$$
\prod_{j=0}^{n-1}\left|1+v_{j+1}\right|^{\sigma_{k j}}=1+\sum_{j=0}^{n-1} \sigma_{k j} v_{j+1}+V_{k}\left(v_{1}, \ldots, v_{n}\right)
$$

где

$$
V_{k}=\prod_{j=0}^{n-1}\left|1+v_{j+1}\right|^{\sigma_{k j}}-1-\sum_{j=0}^{n-1} \sigma_{k j} v_{j+1} .
$$

Здесь

$$
\lim _{\left|v_{1}\right|+\ldots+\left|v_{n}\right| \rightarrow 0} \frac{\partial V_{k}\left(v_{1}, \ldots, v_{n}\right)}{\partial v_{i}}=0, \quad i=\overline{1, n}, \quad V_{k}(0, \ldots, 0)=0 .
$$

Используя (3.17), получаем

$$
\begin{aligned}
& v_{n}^{\prime}=\frac{\beta}{\lambda_{0}-1}\left[f\left(\tau, v_{1}, \ldots, v_{n}\right)-v_{n}+\frac{\sum_{j=0}^{n-1}\left(\sum_{k \in \Gamma} \alpha_{k} c_{k s} \sigma_{k j}\right) v_{j+1}}{\sum_{k \in \Gamma} \alpha_{k} c_{k s}}+\frac{\sum_{k \in \Gamma} \alpha_{k} c_{k s} V_{k}}{\sum_{k \in \Gamma} \alpha_{k} c_{k s}}\right] \\
& v_{n}^{\prime}=\frac{\beta}{\lambda_{0}-1}\left[f\left(\tau, v_{1}, \ldots, v_{n}\right)+\frac{\sum_{j=0}^{n-2}\left(\sum_{k \in \Gamma} \alpha_{k} c_{k s} \sigma_{k j}\right) v_{j+1}}{\sum_{k \in \Gamma} \alpha_{k} c_{k s}}+\right.
\end{aligned}
$$


АСИМПТОТИЧЕСКОЕ ПОВЕДЕНИЕ РЕШЕНИЙ ОБЫКНОВЕННЫХ ДИФФЕРЕНЦИАЛЬНЫХ УРАВНЕНИЙ...

$$
\left.+\left(\frac{\sum_{k \in \Gamma} \alpha_{k} c_{k s} \sigma_{k n-1}}{\sum_{k \in \Gamma} \alpha_{k} c_{k s}}-1\right) v_{n}+V_{n}\left(v_{1}, \ldots, v_{n}\right)\right]
$$

Таким образом, система дифференциальных уравнений (3.13) приобретает вид

$$
\left\{\begin{array}{l}
v_{i}^{\prime}=\frac{\beta}{\lambda_{0}-1}\left[f_{i}\left(\tau, v_{1}, \ldots, v_{n}\right)+a_{0 i}\left(1+v_{i+1}\right)-a_{0 i}\left(1+v_{i}\right)\right], \quad i=\overline{1, n-2}, \\
v_{n-1}^{\prime}=\frac{\beta}{\lambda_{0}-1}\left[f_{n-1}\left(\tau, v_{1}, \ldots, v_{n}\right)+a_{0 n-1}\left(1+v_{n}\right)-a_{0 n-1}\left(1+v_{n-1}\right)\right] \\
v_{n}^{\prime}=\frac{\beta}{\lambda_{0}-1}\left[f_{n}\left(\tau, v_{1}, \ldots, v_{n}\right)+\frac{\sum_{j=0}^{n-2}\left(\sum_{k \in \Gamma} \alpha_{k} c_{k s} \sigma_{k j}\right) v_{j+1}}{\sum_{k \in \Gamma} \alpha_{k} c_{k s}}+\right. \\
\left.\quad+\left(\frac{\sum_{k \in \Gamma} \alpha_{k} c_{k s} \sigma_{k n-1}}{\sum_{k \in \Gamma} \alpha_{k} c_{k s}}-1\right) v_{n}+V_{n}\left(v_{1}, \ldots, v_{n}\right)\right] .
\end{array}\right.
$$

Матрицей коэффициентов, стоящих при линейной части в системе (3.19), является

$$
A=\left(\begin{array}{cccccc}
-a_{01} & a_{01} & 0 & \ldots & 0 & 0 \\
0 & -a_{02} & a_{02} & \ldots & 0 & 0 \\
0 & 0 & -a_{03} & \ldots & 0 & 0 \\
- & - & - & \ldots & - & - \\
0 & 0 & 0 & \ldots & -a_{n-1} & a_{0 n-1} \\
B_{0} & B_{1} & B_{2} & \ldots & B_{n-2} & -a_{0 n}+B_{n-1}
\end{array}\right)
$$

Запишем характеристическое уравнение этой матрицы:

$$
\left|\begin{array}{cccccc}
-a_{01}-\rho & a_{01} & 0 & \ldots & 0 & 0 \\
0 & -a_{02}-\rho & a_{02} & \ldots & 0 & 0 \\
0 & 0 & -a_{03}-\rho & \ldots & 0 & 0 \\
- & - & - & \ldots & - & - \\
0 & 0 & 0 & \ldots & -a_{n-1}-\rho & a_{0 n-1} \\
B_{0} & B_{1} & B_{2} & \ldots & B_{n-2} & -a_{0 n}-\rho+B_{n-1}
\end{array}\right|=0 .
$$

Отсюда следует, что характеристическим уравнением матрицы является уравнение (3.10), которое в силу условий теоремы не имеет корней с нулевой действительной частью.

Ввиду этого факта и (3.16), (3.18) для системы дифференциальных уравнений (3.19) выполнены все условия леммы 2.3. Согласно этой лемме у системы дифференциальных уравнений (3.19) существует хотя бы одно решение $\left(v_{i}\right)_{i=1}^{n}:\left[\tau_{1},+\infty\left[\rightarrow \mathbb{R}^{n}, \tau_{1} \geq \tau_{0}\right.\right.$, стремящееся к нулю при $\tau \rightarrow+\infty$. Более того, когда среди корней характеристического уравнения (3.10) имеется $l$ корней (с учетом кратных), действительные части которых 
имеют знак, противоположный знаку числа $\beta\left(\lambda_{0}-1\right)$, тогда таких решений существует $l$ параметрическое семейство. Каждому такому решению в силу замен (3.12) и условий (3.8) соответствует решение дифференциального уравнения (1.1), допускающее при $t \uparrow \omega$ асимптотические представления

$$
y^{(i-1)}(t)=\nu_{0 n-1}\left|Y_{0}(t)\right|^{1 / s} \frac{\left[\left(\lambda_{0}-1\right) \pi_{\omega}(t)\right]^{n-i}}{\prod_{k=i}^{n-1} a_{0 k}}[1+o(1)], \quad i=1, \ldots, n .
$$

Поскольку в силу условия 2 леммы $2.1 Y_{0}(t) \sim Y(t)$, то данные представления эквивалентны представлениям (3.5).

Теорема 3.2 доказана.

Замечание 3.1. Алгебраическое уравнение (3.10) заведомо не имеет корней с нулевой действительной частью, если $\sum_{m=0}^{n-2}\left|B_{m}\right|<\left|B_{n-1}-1\right|$.

Замечание 3.2. Покажем, что указанные в теореме условия (1.3) заведомо будут выполнены, если справедливы неравенства

$$
\limsup _{t \uparrow \omega} \frac{\ln p_{k}(t)-\ln p_{s}(t)}{|\ln | \pi_{\omega}(t)||}<\frac{\beta}{\lambda_{0}-1} \sum_{j=0}^{n-1}\left(\sigma_{s j}-\sigma_{k j}\right) a_{0 j+1} \quad \text { при всех } \quad k \in\{1, \ldots, m\} \backslash \Gamma .
$$

Действительно, поскольку каждая из функций $\varphi_{k j}, k \in\{1, \ldots, m\}, j \in\{0,1, \ldots, n-1\}$, является правильно меняющейся порядка $\sigma_{k j}$ при $y^{(j)} \rightarrow Y_{j}$ и выражается через медленно меняющуюся, то из определения правильно меняющейся функции следует, что

$$
\varphi_{k j}\left(y^{(j)}(t)\right)=\left|y^{(j)}(t)\right|^{\sigma_{k j}} L_{k j}\left(y^{(j)}(t)\right), \quad k=\overline{1, m}, \quad j=\overline{0, n-1},
$$

где $\left.L_{k j}: \Delta_{Y_{j}} \rightarrow\right] 0,+\infty[, k \in\{1, \ldots, m\}, j \in\{0, n-1\})$, - непрерывная медленно меняющаяся функция при $y^{(j)} \rightarrow Y_{j}$.

Из (3.21) с учетом (2.2) и соотношений (2.5) следует, что

$$
\begin{aligned}
\ln \varphi_{k j}\left(y^{(j)}(t)\right) & =\sigma_{k j} \ln \left|y^{(j)}(t)\right|+\ln L_{k j}\left(y^{(j)}(t)=\ln \left|y^{(j)}(t)\right|\left[\sigma_{k j}+o(1)\right]=\right. \\
& =\frac{a_{0 j+1}}{\lambda_{0}-1} \ln \left|\pi_{\omega}(t)\right|\left[\sigma_{k j}+o(1)\right] \quad k=\overline{1, m}, \quad j=\overline{0, n-1}, \quad \text { при } \quad t \uparrow \omega .
\end{aligned}
$$

Тогда для любого $k \in\{1, \ldots, m\} \backslash \Gamma$

$$
\begin{gathered}
\ln \left[\frac{p_{k}(t) \prod_{j=0}^{n-1} \varphi_{k j}\left(y^{(j)}(t)\right)}{p_{s}(t) \prod_{j=0}^{n-1} \varphi_{s j}\left(y^{(j)}(t)\right)}\right]=\ln \frac{p_{k}(t)}{p_{s}(t)}+\sum_{j=0}^{n-1}\left[\ln \varphi_{k j}\left(y^{(j)}(t)\right)-\ln \varphi_{s j}\left(y^{(j)}(t)\right)\right]= \\
=\ln \frac{p_{k}(t)}{p_{s}(t)}+\frac{\ln \left|\pi_{\omega}(t)\right|}{\lambda_{0}-1} \sum_{j=0}^{n-1}\left[a_{0 j+1}\left(\sigma_{k j}-\sigma_{s j}\right)+o(1)\right]= \\
=\beta \ln \left|\pi_{\omega}(t)\right|\left[\frac{\ln p_{k}(t)-\ln p_{s}(t)}{|\ln | \pi_{\omega}(t)||}+\frac{\beta}{\lambda_{0}-1} \sum_{j=0}^{n-1}\left(\sigma_{k j}-\sigma_{s j}\right) a_{0 j+1}+o(1)\right] \text { при } t \uparrow \omega .
\end{gathered}
$$


Поскольку выражение, стоящее в данном соотношении справа, в силу условий (3.20) стремится к $-\infty$ при $t \uparrow \omega$, отсюда вытекает (1.3).

Замечание 3.3. Заметим, что как только хотя бы для одного $k \in\{1, \ldots, m\} \backslash \Gamma$ выполняется

$$
\lim _{t \uparrow \omega} \inf \frac{\ln p_{k}(t)-\ln p_{s}(t)}{|\ln | \pi_{\omega}(t)||}>\frac{\beta}{\lambda_{0}-1} \sum_{j=0}^{n-1}\left(\sigma_{s j}-\sigma_{k j}\right) a_{0 j+1},
$$

то, повторяя рассуждения замечания 3.2 , легко показать, что

$$
\lim _{t \uparrow \omega} \frac{p_{k}(t) \prod_{j=0}^{n-1} \varphi_{k j}\left(y^{(j)}(t)\right)}{p_{s}(t) \prod_{j=0}^{n-1} \varphi_{s j}\left(y^{(j)}(t)\right)}=+\infty,
$$

и поэтому условия (1.3) не выполняются.

Для формулировки следующего результата введем вспомогательные обозначения, полагая

$$
\begin{gathered}
J_{k 0}(t)=\int_{A_{k 0}}^{t} p_{k}(s) d s, \quad J_{k 00}(t)=\int_{A_{k 00}}^{t} J_{k 0}(s) d s, \quad k=\overline{1, m}, \\
J_{k n}(t)=\int_{A_{k n}}^{t} p_{k}(s)\left|\pi_{\omega}(s)\right|^{\mu_{k n}} \prod_{j=0}^{n-2} L_{k j}\left(\nu_{0 j}\left|\pi_{\omega}(s)\right|^{n-j-1}\right) d s, \quad k=\overline{1, m},
\end{gathered}
$$

где каждый из пределов интегрирования $A_{k m}, A_{k m m}, m \in\{0,1\}$, выбирается равным точке $a_{0} \in\left[a, \omega\left[\right.\right.$ (справа от которой, т. е. при $t \in\left[a_{0}, \omega[\right.$, подынтегральная функция непрерывна), если при этом значении предела интегрирования соответствующий интеграл стремится к $\pm \infty$ при $t \uparrow \omega$, и равным $\omega$, если при таком значении предела интегрирования он стремится к нулю при $t \uparrow \omega$.

Теорема 3.3. Пусть для некоторого $s \in\{1, \ldots, m\}$ выполняется неравенство $\gamma_{s} \neq 0$ и для непустого множества $\Gamma \subset\{1, \ldots, m\}$ медленно меняющиеся составляющие $L_{k j}(y)$ функций $\varphi_{k j}$ удовлетворяют условию $S$ для каждого $k \in \Gamma, j \in\{0, \ldots, n-1\}$. Пусть, кроме того, существует непрерывная функция $b_{s}:[a, \omega[\longrightarrow \mathbb{R} \backslash\{0\}$ такая, что

$$
\lim _{t \uparrow \omega}\left|\pi_{\omega}(t) b_{s}(t)\right|=+\infty
$$

Тогда для существования $P_{\omega}\left(Y_{0}, \ldots, Y_{n-1}, 1\right)$-решения дифференциального уравнения (1.1), для которого выполняются условия (1.3), (1.4), $\sum_{k \in \Gamma} \alpha_{k} c_{k s} \neq 0 u$

$$
\frac{y^{\prime}(t)}{y(t)} \sim b_{s}(t) \quad \text { npu } \quad t \uparrow \omega,
$$

где $b_{s}:[a, \omega[\rightarrow \mathbb{R} \backslash\{0\}-$ непрерывная функция такая, что

$$
\lim _{t \uparrow \omega}\left|\pi_{\omega}(t) b_{s}(t)\right|=+\infty
$$

ISSN 1562-3076. Нелінійні коливання, 2018, m. 21, № 2 
необходимо, а если алгебрачческое относительно р уравнение

$$
(1+\rho)^{n}=\sum_{j=0}^{n-1} \sigma_{s j}(1+\rho)^{j}
$$

не имеет корней с нулевой действительной частью, то и достаточно, чтобы

$$
b_{s}(t) \sim \frac{p_{s}(t)}{\gamma_{s} J_{s 0}(t)}, \quad \frac{p_{s}(t)}{J_{s 0}(t)} \sim \frac{J_{s 0}(t)}{J_{s 00}(t)} \quad n p u \quad t \uparrow \omega, \quad \nu_{0 j} \lim _{t \uparrow \omega}\left|J_{s 0}(t)\right|^{1 / \gamma_{s}}=Y_{j}, \quad j=\overline{0, n-1},
$$

выполнялись неравенства (1.6) и следующие неравенства:

$$
\left.\sum_{k \in \Gamma} \alpha_{k} c_{k s} \nu_{0 n-1} \gamma_{s} J_{s 0}(t)>0, \quad \nu_{0 j} \nu_{0 n-1}\left(\gamma_{s} J_{s 0}(t)\right)^{n-j-1}>0, \quad j=\overline{0, n-2}, \quad \text { npu } \quad t \in\right] a, \omega[.
$$

Более того, для каждого такого решения при $t \uparrow \omega$ имеют место асимптотические представления

$$
\begin{gathered}
y^{(j)}(t)=\left(\frac{\gamma_{s} J_{s 00}(t)}{J_{s 0}(t)}\right)^{n-j-1} y^{(n-1)}(t)[1+o(1)], \quad j=\overline{0, n-2}, \\
\frac{\left|y^{(n-1)}(t)\right|^{\gamma_{s}}}{\prod_{j=0}^{n-1} L_{s j}\left(\left(\frac{\gamma_{s} J_{s o 0}(t)}{J_{s 0}(t)}\right)^{n-j-1} y^{(n-1)}(t)\right)} \\
=\left(\sum_{k \in \Gamma} \alpha_{k} c_{k s}\right) \nu_{0 n-1} \gamma_{s} J_{s 0}(t)\left|\frac{\gamma_{s} J_{s 00}(t)}{J_{s 00}(t)}\right|^{\mu_{s n}}[1+o(1)],
\end{gathered}
$$

причем решений с такими представлениями существует целое l-параметрическое семейство, если среди корней алгебраического уравнения (3.25) имеется l (с учетом кратных) корней, действительные части которых имеют знак, противоположный знаку $\nu_{0 n-1} \sum_{k \in \Gamma} \alpha_{k} c_{k s}$.

Доказательство. Необходимость. Пусть $y:\left[t_{0}, \omega\left[\longrightarrow \mathbb{R}-\right.\right.$ произвольное $P_{\omega}\left(Y_{0}, \ldots\right.$ $\left.\ldots, Y_{n-1}, 1\right)$-решение дифференциального уравнения (1.1), для которого наряду с (1.3), (1.4) и $\sum_{k \in \Gamma} \alpha_{k} c_{k s} \neq 0$ выполняется условие (3.23). Тогда, как было указано ранее, справедливы неравенства (1.6) и в силу леммы 2.2, а также из условий (3.24) имеем

$$
\frac{y^{(k)}(t)}{y^{(k-1)}(t)} \sim b_{s}(t) \quad \text { при } \quad t \uparrow \omega(k=\overline{1, n}) \quad \text { и } \quad \beta \beta_{s} \lim _{t \uparrow \omega} \frac{\pi_{\omega}(t) y^{\prime}(t)}{y(t)}=+\infty .
$$

Отсюда, в частности, следует, что

$$
\ln \left|y^{(k-1)}(t)\right| \sim \int_{a}^{t} b_{s}(\tau) d \tau \rightarrow \pm \infty, \quad k=\overline{1, n}, \quad \text { при } \quad t \uparrow \omega .
$$


В силу условий (1.3) и (1.4) из (1.1) следует, что для данного решения имеет место асимптотическое соотношение

$$
y^{(n)}(t)=\left(\sum_{k \in \Gamma} \alpha_{k} c_{k s}\right) p_{s}(t)[1+o(1)] \prod_{j=0}^{n-1} \varphi_{s j}\left(y^{(j)}(t)\right) \quad \text { при } \quad t \uparrow \omega .
$$

Согласно лемме 2.1 существуют непрерывно дифференцируемые правильно меняющиеся при $y^{(j)} \rightarrow Y_{j}$ функции $\left.\varphi_{0 s j}: \Delta_{Y_{j}} \longrightarrow\right] 0,+\infty\left[\right.$ порядков $\sigma_{s j}, j=\overline{0, n-1}$, такие, чTO

$$
\varphi_{s j}\left(y^{(j)}\right) \sim \varphi_{0 s j}\left(y^{(j)}\right) \quad \text { при } \quad t \uparrow \omega, \quad \lim _{\substack{y(j) \rightarrow Y_{j} \\ y(j) \in \Delta_{Y_{j}}}} \frac{y^{(j)} \varphi_{0 s j}^{\prime}\left(y^{(j)}\right)}{\varphi_{0 s j}\left(y^{(j)}\right)}=\sigma_{s j}, \quad j=\overline{0, n-1} .
$$

В силу (3.33) и (3.30) имеем

$$
\begin{aligned}
\left(\frac{y^{(k-1)}(t)}{\prod_{j=0}^{n-1} \varphi_{0 s j}\left(y^{(j)}(t)\right)}\right)^{\prime}= & \frac{y^{(k)}(t)}{\prod_{j=0}^{n-1} \varphi_{0 s j}\left(y^{(j)}(t)\right)} \\
& \times\left[1-\sum_{j=0}^{n-1} \frac{y^{(k-1)}(t) y^{(j+1)}(t)}{y^{(k)}(t) y^{(j)}(t)} \frac{y^{(j)}(t) \varphi_{0 s j}^{\prime}\left(y^{(j)}(t)\right)}{\varphi_{0 s j}\left(y^{(j)}(t)\right)}\right]= \\
= & \frac{y^{(k)}(t)}{\prod_{j=0}^{n-1} \varphi_{0 s j}\left(y^{(j)}(t)\right)}\left[\gamma_{s}+o(1)\right], \quad k=1, \ldots, n \quad \text { при } t \uparrow \omega .
\end{aligned}
$$

Поэтому соотношение (3.32) может быть переписано в виде

$$
\begin{aligned}
\left(\frac{y^{(n-1)}(t)}{\prod_{j=0}^{n-1} \varphi_{0 s j}\left(y^{(j)}(t)\right)}\right)^{\prime} & =\frac{y^{(n)}(t)}{\prod_{j=0}^{n-1} \varphi_{0 s j}\left(y^{(j)}(t)\right)}\left[\gamma_{s}+o(1)\right]= \\
& =\left(\sum_{k \in \Gamma} \alpha_{k} c_{k s}\right) \gamma_{s} p_{s}(t)[1+o(1)] \quad \text { при } t \uparrow \omega .
\end{aligned}
$$

Интегрируя это соотношение на промежутке от $t_{1}$ до $t$, получаем

$$
\frac{y^{(n-1)}(t)}{\prod_{j=0}^{n-1} \varphi_{0 s j}\left(y^{(j)}(t)\right)}=C+\left(\sum_{k \in \Gamma} \alpha_{k} c_{k s}\right) \gamma_{s} J_{s 0}(t)[1+o(1)] \quad \text { при } \quad t \uparrow \omega
$$

где $C$ - некоторая вещественная постоянная.

В случае, когда в функции $J_{s 0}$ предел интегрирования $A_{s 0}=a$, тогда $J_{s 0}(t) \rightarrow+\infty$ при $t \uparrow \omega$ и полученное соотношение представимо в виде

$$
\frac{y^{(n-1)}(t)}{\prod_{j=0}^{n-1} \varphi_{0 s j}\left(y^{(j)}(t)\right)}=\left(\sum_{k \in \Gamma} \alpha_{k} c_{k s}\right) \gamma_{s} J_{s 0}(t)[1+o(1)] \quad \text { при } \quad t \uparrow \omega .
$$


Покажем, что в случае $A_{s 0}=\omega$, когда $J_{s 0}(t) \rightarrow 0$ при $t \uparrow \omega$, постоянная $C=0$. Предположим противное, т. е. что в этом случае $C \neq 0$. Тогда

$$
\frac{y^{(n-1)}(t)}{\prod_{j=0}^{n-1} \varphi_{0 s j}\left(y^{(j)}(t)\right)}=C+o(1) \quad \text { при } \quad t \uparrow \omega,
$$

но этого быть не может, поскольку в силу (3.31)

$$
\ln \left|\frac{y^{(n-1)}(t)}{\prod_{j=0}^{n-1} \varphi_{0 s j}\left(y^{(j)}(t)\right)}\right|=\int_{a}^{t} b_{s}(\tau) d \tau\left[\gamma_{s}+o(1)\right] \rightarrow \pm \infty \quad \text { при } \quad t \uparrow \omega .
$$

Значит, при $A_{s 0}=\omega$ также имеет место представление (3.35).

Аналогично из (3.35) с использованием (3.34) при $k=n-1$ получаем

$$
\frac{y^{(n-2)}(t)}{\prod_{j=0}^{n-1} \varphi_{0 s j}\left(y^{(j)}(t)\right)}=\left(\sum_{k \in \Gamma} \alpha_{k} c_{k s}\right) \gamma_{s}^{2} J_{s 00}(t)[1+o(1)] \quad \text { при } \quad t \uparrow \omega .
$$

Из (3.32), (3.35) и (3.36) с учетом (3.33) имеем

$$
\frac{y^{(n)}(t)}{y^{(n-1)}(t)}=\frac{p_{s}(t)}{\gamma_{s} J_{s 0}(t)}[1+o(1)], \quad \frac{y^{(n-1)}(t)}{y^{(n-2)}(t)}=\frac{J_{s 0}(t)}{\gamma_{s} J_{s 00}(t)}[1+o(1)] \quad \text { при } \quad t \uparrow \omega .
$$

Поэтому в силу (2.6) и (3.23) выполняется условия (3.26) и ввиду тождеств

$$
y^{(j)}(t)=\frac{y^{(j)}(t)}{y^{(j+1)}(t)} \cdots \frac{y^{(n-2)}(t)}{y^{(n-1)}(t)} y^{(n-1)}(t), \quad j=\overline{0, n-2},
$$

имеют место асимптотические представления (3.28). Кроме того, из (3.35) и (3.28) следует, что выполняются неравенства (3.27).

Используя теперь приведенные выше тождества, представления (3.28) и лемму 2.1, находим

$$
\begin{aligned}
\varphi_{0 s j}\left(y^{(j)}(t)\right)= & \left|y^{(j)}(t)\right|^{\sigma_{s j}} L_{0 s j}\left(y^{(j)}(t)\right) \sim \\
\sim & \left|\left(\frac{\gamma_{s} J_{s 00}(t)}{J_{s 0}(t)}\right)^{n-j-1} y^{(n-1)}(t)\right|^{\sigma_{s j}} \times \\
& \times L_{0 s j}\left(\left(\frac{\gamma_{s} J_{s 00}(t)}{J_{s 0}(t)}\right)^{n-j-1} y^{(n-1)}(t)[1+o(1)]\right) \sim \\
& \sim\left|\frac{\gamma_{s} J_{s 00}(t)}{J_{s 0}(t)}\right|^{(n-j-1) \sigma_{s j}}\left|y^{(n-1)}(t)\right|^{\sigma_{s j}} \times \\
& \times L_{0 s j}\left(\left(\frac{\gamma_{s} J_{s 00}(t)}{J_{s 0}(t)}\right)^{n-j-1} y^{(n-1)}(t)\right), \quad j=\overline{0, n-1} \quad \text { при } \quad t \uparrow \omega .
\end{aligned}
$$


Ввиду этих соотношений из (3.35) получаем представление

$$
\begin{gathered}
\frac{\left|y^{n-1}(t)\right|^{\gamma_{s}}\left|\frac{J_{s 0}(t)}{\gamma_{s} J_{s 00}(t)}\right|^{\mu_{s n}}}{\prod_{j=0}^{n-1} L_{0 s j}\left(\left(\frac{\gamma_{s} J_{s 00}(t)}{J_{s 0}(t)}\right)^{n-j-1} y^{(n-1)}(t)\right)}= \\
=\left(\sum_{k \in \Gamma} \alpha_{k} c_{k s}\right) \nu_{0 n-1} \gamma_{s} J_{s 0}(t)[1+o(1)] \quad \text { при } t \uparrow \omega,
\end{gathered}
$$

из которого с учетом первых из соотношений (2.1) вытекает представление (3.29).

Достаточность. Пусть соблюдаются условия (1.6), (3.23), (3.26), (3.27) и алгебраическое уравнение (3.25) не имеет корней с нулевой действительной частью.

Покажем, что в данном случае существуют $P_{\omega}\left(Y_{0}, \ldots, Y_{n-1}, 1\right)$-решения уравнения (1.1), допускающие при $t \uparrow \omega$ асимптотические представления (3.28), (3.29) и выясним вопрос о количестве таких решений.

Сначала рассмотрим соотношение

$$
\frac{|Y|^{\gamma_{s}}}{\prod_{j=0}^{n-1} L_{0 s j}\left(\left(\frac{\gamma_{s} J_{s 00}(t)}{J_{s 0}(t)}\right)^{n-j-1} Y\right)}=Q_{s}(t)\left(1+v_{n}\right),
$$

где $\left.L_{0 s j}: \Delta_{Y_{j}} \longrightarrow\right] 0,+\infty[, j=\overline{0, n-1},-$ непрерывно дифференцируемые медленно меняющиеся при $y^{(j)} \rightarrow Y_{j}$ функции, удовлетворяющие условиям (2.1) (при $k=s$ ) и существующие в силу леммы 2.1, а также

$$
Q_{s}(t)=\left(\sum_{k \in \Gamma} \alpha_{k} c_{k s}\right) \nu_{0 n-1} \gamma_{s} J_{s 0}(t)\left|\frac{\gamma_{s} J_{s 00}(t)}{J_{s 0}(t)}\right|^{\mu_{s n}} .
$$

Положим

$$
d=\frac{1}{2\left|\gamma_{s}\right|}, \quad \mathbb{R}_{d}=\{z \in \mathbb{R}:|z| \leq d\}, \quad \mathbb{R}_{1 / 2}=\left\{v_{n} \in \mathbb{R}:\left|v_{n}\right| \leq \frac{1}{2}\right\}
$$

и покажем, что соотношение (3.37) однозначно определяет заданную на множестве $\left[t_{0}, \omega\left[\times \mathbb{R}_{1 / 2}, t_{0} \in\left[a, \omega\left[\right.\right.\right.\right.$, непрерывно дифференцируемую неявную функцию $Y=Y\left(t, v_{n}\right)$ вида

$$
Y\left(t, v_{n}\right)=\nu_{0 n-1}\left|J_{s 0}(t)\right|^{\frac{1}{\gamma_{s}}+z\left(t, v_{n}\right)},
$$

где функция $z$ такова, что

$$
\left|z\left(t, v_{n}\right)\right| \leq d \quad \text { при } \quad\left(t, v_{n}\right) \in\left[t_{0}, \omega\left[\times \mathbb{R}_{1 / 2}\right.\right.
$$

И

$$
\lim _{t \uparrow \omega} z\left(t, v_{n}\right)=0 \quad \text { равномерно по } \quad v_{n} \in \mathbb{R}_{1 / 2} \text {. }
$$

ISSN 1562-3076. Нелінійні коливання, 2018, m. 21, № 2 
Полагая в (3.37)

$$
Y=\nu_{o n-1}\left|J_{s 0}(t)\right|^{\frac{1}{\gamma_{s}}+z}
$$

и затем логарифмируя полученное при этом соотношение, после элементарных преобразований находим, что

$$
z=a(t)+b\left(t, v_{n}\right)+Z(t, z)
$$

где

$$
\begin{gathered}
a(t)=\frac{1}{\gamma_{s}}\left(\frac{\ln Q_{s}(t)}{\ln \left|J_{s 0}(t)\right|}-1\right), \quad b\left(t, v_{n}\right)=\frac{\ln \left(1+v_{n}\right)}{\gamma_{s} \ln \left|J_{s 0}(t)\right|}, \\
Z(t, z)=\frac{1}{\gamma_{s} \ln \left|J_{s o}(t)\right|} \sum_{j=0}^{n-1} \ln L_{0 s}\left(\nu_{0 n-1}\left(\frac{\gamma_{s} J_{s 00}(t)}{J_{s 0}(t)}\right)^{n-j-1}\left|J_{s 0}(t)\right|^{\frac{1}{\gamma_{s}}+z}\right) .
\end{gathered}
$$

В силу последних из неравенств (3.27), а также второго и третьего из условий (3.26)

$$
\begin{aligned}
& \nu_{0 n-1} \lim _{t \uparrow \omega}\left(\frac{\gamma_{s} J_{s 00}(t)}{J_{s 0}(t)}\right)^{n-j-1}\left|J_{s 0}(t)\right|^{\frac{1}{\gamma_{s}}+z}=\nu_{0 j} \lim _{t \uparrow \omega}\left|\frac{\gamma_{s} J_{s 00}(t)}{J_{s 0}(t)}\right|^{n-j-1}\left|J_{s 0}(t)\right|^{\frac{1}{\gamma_{s}}+z}= \\
& =\nu_{o j} \lim _{t \uparrow \omega} \exp \left(\ln \left|J_{s 0}(t)\right|\left[\frac{1}{\gamma_{s}}+z+(n-j-1)\left(\frac{\ln \left|\gamma_{s} J_{s 00}(t)\right|}{\ln \left|J_{s 0}(t)\right|}-1\right)\right]\right)= \\
& =\nu_{o j} \lim _{t \uparrow \omega} \exp \left(\ln \left|J_{s 0}(t)\right|\left[\frac{1}{\gamma_{s}}+z+o(1)\right]\right)= \\
& =\nu_{0 j} \lim _{t \uparrow \omega}\left|J_{s 0}(t)\right|^{1 / \gamma_{s}}=Y_{j}, \quad j=\overline{0, n-1} \quad \text { при }|z| \leq d .
\end{aligned}
$$

Поэтому правая часть в (3.40) непрерывно дифференцируема на множестве $\left[t_{0}, \omega\left[\times \mathbb{R}_{1 / 2} \times\right.\right.$ $\times \mathbb{R}_{d}$, где $t_{0}$ - некоторое число на промежутке $[a, \omega[$.

Кроме того, в силу второго и третьего из условий (3.26), а также вторых из условий (1.6) имеем

$$
\begin{gathered}
\lim _{t \uparrow \omega} a(t)=0, \quad \lim _{t \uparrow \omega} b\left(t, v_{n}\right)=0 \quad \text { равномерно по } \quad v_{n} \in \mathbb{R}_{1 / 2}, \\
\lim _{t \uparrow \omega} Z(t, z)=0, \quad \lim _{t \uparrow \omega} \frac{\partial Z(t, z)}{\partial z}=0 \quad \text { равномерно по } \quad z \in \mathbb{R}_{d} .
\end{gathered}
$$

Согласно этим условиям существует число $t_{2} \in\left[t_{1}, \omega\left[\right.\right.$ такое, что на множестве $\left[t_{2}, \omega\left[\times \mathbb{R}_{1 / 2} \times\right.\right.$ $\times \mathbb{R}_{d}$ выполняется неравенство

$$
\left|a(t)+b\left(t, v_{1}, v_{2}\right)+Z(t, z)\right| \leq d
$$

и условие Липшица

$$
\left|Z\left(t, z_{1}\right)-Z\left(t, z_{2}\right)\right| \leq \frac{1}{2}\left|z_{1}-z_{2}\right| \quad \text { при } \quad t \in\left[t_{2}, \omega\left[\quad \text { и } \quad z_{1}, z_{2} \in \mathbb{R}_{d} .\right.\right.
$$


Аналогично работе [2] заключаем, что в силу замены (3.39) полученной функции $z$ соответствует непрерывно дифференцируемая на множестве $\left[t_{0}, \omega\left[\times \mathbb{R}_{1 / 2}\right.\right.$ функция $Y$ вида (3.38), которая является решением уравнения (3.37) и удовлетворяет условиям

$$
\begin{gathered}
\nu_{0 j}\left(\frac{\gamma_{s} J_{s 00}(t)}{J_{s 0}(t)}\right)^{n-j-1} Y\left(t, v_{n}\right) \in \Delta_{Y_{j}}, \quad j=\overline{0, n-1} \quad \text { при } \quad\left(t, v_{n}\right) \in\left[t_{0}, \omega\left[\times \mathbb{R}_{1 / 2},\right.\right. \\
\nu_{0 j} \lim _{t \uparrow \omega}\left(\frac{\gamma_{s} J_{s 00}(t)}{J_{s 0}(t)}\right)^{n-j-1} Y\left(t, v_{n}\right)=Y_{j}, \quad j=\overline{0, n-1}, \quad \text { равномерно по } \quad v_{n} \in \mathbb{R}_{1 / 2} .
\end{gathered}
$$

Теперь, применяя к дифференциальному уравнению (1.1) преобразование

$$
\begin{gathered}
\frac{y^{(j)}(t)}{y^{(n-1)}(t)}=\left(\frac{\gamma_{s} J_{s 00}(t)}{J_{s 0}(t)}\right)^{n-j-1}\left[1+v_{j+1}(\tau)\right], \quad j=\overline{0, n-1}, \\
y^{(n-1)}(t)=Y\left(t, v_{n}(\tau)\right), \quad \tau=\left(\sum_{k \in \Gamma} \alpha_{k} c_{k s}\right) \nu_{0 n-1} \ln \left|J_{s 00}(t)\right|^{1 / \gamma_{s}}
\end{gathered}
$$

и учитывая, что функция $y^{(n-1)}(t)=Y\left(t, v_{n}(\tau)\right)$ при $t \in\left[t_{0}, \omega\left[\right.\right.$ и $v_{n}(\tau) \in \mathbb{R}^{1 / 2}$ удовлетворяет уравнению

$$
\frac{\left|y^{(n-1)}(t)\right|^{\gamma_{s}}}{\prod_{j=0}^{n-1} L_{0 s j}\left(\left(\frac{\gamma_{s} J_{s 00}(t)}{J_{s 0}(t)}\right)^{n-j-1} y^{(n-1)}(t)\right)}=Q_{s}(t)\left[1+v_{n}(\tau)\right]
$$

получаем систему дифференциальных уравнений вида

$$
\left\{\begin{aligned}
v_{i}^{\prime}= & \frac{\nu_{0 n-1}}{\left(\sum_{k \in \Gamma} \alpha_{k} c_{k s}\right)}\left(1+v_{i+1}-(n-j-1) \gamma_{s}[1-h(\tau)]\left(1+v_{i}\right)-\right. \\
& \left.-h(\tau) \frac{\left(1+v_{i}\right) \prod_{j=0}^{n-2}\left|1+v_{j+1}\right|^{\sigma_{s j}}}{1+v_{n}} G\left(\tau, v_{1}, \ldots, v_{n}\right)\right), \quad i=\overline{1, n-2}, \\
v_{n-1}^{\prime}= & \frac{\nu_{0 n-1}}{\left(\sum_{k \in \Gamma} \alpha_{k} c_{k s}\right)}\left(1-\gamma_{s}[1-h(\tau)]\left(1+v_{n-1}\right)-\right. \\
& \left.-h(\tau) \frac{\left(1+v_{n-1}\right) \prod_{j=0}^{n-2}\left|1+v_{j+1}\right|^{\sigma_{s j}}}{1+v_{n}} G\left(\tau, v_{1}, \ldots, v_{n}\right)\right) \\
v_{n}^{\prime}= & \frac{\nu_{0 n-1}}{\left(\sum_{k \in \Gamma} \alpha_{k} c_{k s}\right)}\left(\gamma_{s} h(\tau) \prod_{j=0}^{n-2}\left|1+v_{j+1}\right|^{\sigma_{s j}} G\left(\tau, v_{1}, \ldots, v_{n}\right)-\right. \\
& \left.-H\left(\tau, v_{1}, \ldots, v_{n}\right)\left(1+v_{n}\right)-\gamma_{s} q(\tau)\left(1+v_{n}\right)\right)
\end{aligned}\right.
$$

в которой

$$
h(\tau(t))=\frac{p_{s}(t) J_{s 00}(t)}{J_{s 0}^{2}(t)}, \quad q(\tau)=h(\tau)+\mu_{s n}[1-h(\tau)]
$$

ISSN 1562-3076. Нелінійні коливання, 2018, m. 21, № 2 
$G\left(\tau(t), v_{1}, \ldots, v_{n}\right)=$

$$
\begin{gathered}
=\frac{L_{s n-1}\left(Y\left(t, v_{n}\right)\right) \prod_{j=0}^{n-2} L_{s j}\left(\left(\frac{\gamma_{s} J_{s 00}(t)}{J_{s 0}(t)}\right)^{n-j-1} Y\left(t, v_{n}\right)\left(1+v_{j+1}\right)\right)}{\left.\prod_{j=0}^{n-1} L_{0 s j}\left(\left(\frac{\gamma_{s} J_{s 00}(t)}{J_{s 0}(t)}\right)^{n-j-1} Y\left(t, v_{n}\right)\right)\right)} \times \\
\times \frac{\sum_{k=1}^{m} \alpha_{k} p_{k}(t) \varphi_{k n-1}\left(Y\left(t, v_{n}\right)\right) \prod_{j=0}^{n-2} \varphi_{k j}\left(\left(\frac{\gamma_{s} J_{s 00}(t)}{J_{s 0}(t)}\right)^{n-j-1} Y\left(t, v_{n}\right)\left(1+v_{j+1}\right)\right)}{\left(\sum_{k \in \Gamma} \alpha_{k} c_{k s}\right) p_{s}(t) \varphi_{s n-1}\left(Y\left(t, v_{n}\right)\right) \prod_{j=0}^{n-2} \varphi_{s j}\left(\left(\frac{\gamma_{s} J_{s 00}(t)}{J_{s 0}(t)}\right)^{n-j-1} Y\left(t, v_{n}\right)\left(1+v_{j+1}\right)\right)}, \\
H\left(\tau(t), v_{1}, \ldots, v_{n}\right)=\sum_{j=0}^{n-1} \frac{\left(\frac{\gamma_{s} J_{s 00}(t)}{J_{s 0}(t)}\right)^{n-j-1} Y\left(t, v_{n}\right) L_{0 s j}^{\prime}\left(\left(\frac{\gamma_{s} J_{s 00}(t)}{J_{s 0}(t)}\right)^{n-j-1} Y\left(t, v_{n}\right)\right)}{L_{0 s j}\left(\left(\frac{\gamma_{s} J_{s 00}(t)}{J_{s 0}(t)}\right)^{n-j-1} Y\left(t, v_{n}\right)\right)} \times \\
\times\left(\gamma_{s}(n-j-1)[1-h(\tau(t))]+h(\tau) \frac{\prod_{j=0}^{n-2}\left|1+v_{j+1}\right|^{\sigma_{s j}}}{1+v_{n}} G\left(\tau, v_{1}, \ldots, v_{n}\right)\right) .
\end{gathered}
$$

В силу (3.26) и (3.27) функция

$$
\tau(t)=\left(\sum_{k \in \Gamma} \alpha_{k} c_{k s}\right) \nu_{0 n-1} \ln \left|J_{s 00}(t)\right|^{1 / \gamma_{s}}
$$

имеет свойства

$$
\tau^{\prime}(t)>0 \quad \text { при } \quad t \in\left[t_{0}, \omega\left[, \quad \lim _{t \uparrow \omega} \tau(t)=+\infty\right.\right.
$$

и существует $t_{1} \in\left[t_{0}, \omega[\right.$ такое, что на множестве

$$
\left[\tau_{1},+\infty\left[\times \mathbb{R}_{1 / 2}^{n}, \quad \text { где } \quad \tau_{1}=\tau\left(t_{1}\right), \quad \mathbb{R}_{1 / 2}^{n}=\left\{\left(v_{1}, \ldots, v_{n}\right) \in \mathbb{R}^{n}:\left|v_{i}\right| \leq \frac{1}{2}(i=\overline{1, n})\right\}\right.\right.
$$

правые части системы уравнений (3.47) непрерывны. Согласно второму из условий (3.26) имеем

$$
\lim _{\tau \rightarrow+\infty} h(\tau)=\lim _{t \uparrow \omega} h(\tau(t))=1, \quad \lim _{\tau \rightarrow+\infty} q(\tau)=1 .
$$

Далее, используя условия (3.45), показываем, что вторая дробь в представлении функции $G$ стремится к единице при $t \uparrow \omega$ равномерно по $\left(v_{1}, \ldots, v_{n}\right) \in \mathbb{R}_{1 / 2}^{n}$. Кроме того, в силу условий (3.45), леммы 2.1, условий (1.6) и (3.48) равномерно по $\left(v_{1}, \ldots, v_{n}\right) \in \mathbb{R}_{1 / 2}^{n}$ первая дробь в представлении функции $G$ стремится к единице и функция $H$ стремится к нулю 
при $t \uparrow \omega$. Поэтому система дифференциальных уравнений (3.47) может быть записана в виде

$$
\left\{\begin{array}{l}
v_{i}^{\prime}=\frac{\nu_{0 n-1}}{\left(\sum_{k \in \Gamma} \alpha_{k} c_{k s}\right)}\left(f_{i}\left(\tau, v_{1}, \ldots, v_{n}\right)+1+v_{i+1}-\frac{\left(1+v_{i}\right) \prod_{j=0}^{n-2}\left|1+v_{j+1}\right|^{\sigma_{s j}}}{1+v_{n}}\right), \\
i=\overline{1, n-2}, \\
v_{n-1}^{\prime}=\frac{\nu_{0 n-1}}{\left(\sum_{k \in \Gamma} \alpha_{k} c_{k s}\right)}\left(f_{n-1}\left(\tau, v_{1}, \ldots, v_{n}\right)+1-\frac{\left(1+v_{n-1}\right) \prod_{j=0}^{n-2}\left|1+v_{j+1}\right|^{\sigma_{s j}}}{1+v_{n}}\right), \\
v_{n}^{\prime}=\frac{\nu_{0 n-1}}{\left(\sum_{k \in \Gamma} \alpha_{k} c_{k s}\right)}\left(f_{n}\left(\tau, v_{1}, \ldots, v_{n}\right)+\gamma_{s} \prod_{j=0}^{n-2}\left|1+v_{j+1}\right|^{\sigma_{s j}}-\gamma_{s}\left(1+v_{n}\right)\right),
\end{array}\right.
$$

где

$$
\lim _{\tau \rightarrow+\infty} f_{i}\left(\tau, v_{1}, \ldots, v_{n}\right)=0, \quad i=\overline{1, n}, \quad \text { равномерно по } \quad\left(v_{1}, \ldots, v_{n}\right) \in \mathbb{R}_{1 / 2}^{n} .
$$

Далее, выделяя линейные части в слагаемых, стоящих после функций $f_{i}, i=\overline{1, n}$, получаем систему дифференциальных уравнений

$$
v_{i}^{\prime}=\frac{\nu_{0 n-1}}{\left(\sum_{k \in \Gamma} \alpha_{k} c_{k s}\right)}\left(f_{i}\left(\tau, v_{1}, \ldots, v_{n}\right)+\sum_{k=1}^{n} p_{i k} v_{k}+V_{i}\left(v_{1}, \ldots, v_{n}\right)\right), \quad i=\overline{1, n},
$$

в которой

$$
\begin{gathered}
p_{i i}=-1-\sigma_{s i-1}, \quad p_{i i+1}=1-\sigma_{s i}, \quad p_{i k}=-\sigma_{s k-1} \\
\text { при } k \neq i, i+1, n \quad p_{i n}=1, \quad i=\overline{1, n-2}, \\
p_{n-1 k}=-\sigma_{s k-1} \quad \text { при } \quad k=\overline{1, n-2}, \\
p_{n-1 n-1}=-1-\sigma_{s n-2}, \quad p_{n-1 n}=1, \\
p_{n k}=\gamma_{s} \sigma_{s k-1} \quad \text { при } \quad k=\overline{1, n-1}, \quad p_{n n}=-\gamma_{s}, \\
V_{i}\left(v_{1}, \ldots, v_{n}\right)=-\frac{\left(1+v_{i}\right) \prod_{j=0}^{n-2}\left|1+v_{j+1}\right|^{\sigma_{s j}}}{1+v_{n}}-v_{n}+ \\
+\left(1+\sigma_{s i-1}\right) v_{i}+\sum_{\substack{k=1 \\
k \neq i}}^{n-1} \sigma_{s k-1} v_{k}, \quad i=\overline{1, n-1}, \\
V_{n}\left(v_{1}, \ldots, v_{n}\right)=\gamma_{s} \prod_{j=0}^{n-2}\left|1+v_{j+1}\right|^{\sigma_{s j}}-\gamma_{s} \sum_{k=1}^{n-1} \sigma_{s k-1} v_{k} .
\end{gathered}
$$

ISSN 1562-3076. Нелінійні коливання, 2018, m. 21, № 2 
Здесь

$$
\lim _{\left|v_{1}\right|+\cdots+\left|v_{n}\right| \rightarrow 0} \frac{\partial V_{i}\left(v_{1}, \ldots, v_{n}\right)}{\partial v_{k}}=0, \quad i, k=\overline{1, n}
$$

характеристическое уравнение $\operatorname{det}[P-\rho E]=0$, где $P=\left(p_{i k}\right)_{i, k=1}^{n}$ и $E-$ единичная матрица размерности $n \times n$, имеет вид (3.35). В силу условий теоремы это уравнение не имеет корней с нулевой действительной частью. Тем самым показано, что для системы (3.49) выполнены все условия леммы 2.3. Согласно этой лемме данная система имеет по крайней мере одно решение $\left(v_{i}\right)_{i=1}^{n}:\left[\tau_{2},+\infty\left[\rightarrow \mathbb{R}^{n}\right.\right.$, где $\tau_{2} \geq \tau_{1}$, стремящееся к нулю при $\tau \rightarrow+\infty$, причем таких решений существует $l$-параметрическое семейство, если среди корней алгебраического уравнения (3.35) имеется $l$ корней (с учетом кратных), действительные части которых имеют знак, противоположный знаку числа $\alpha_{s} \nu_{0 n-1}$. Каждому такому решению, в силу замены (3.46) и первых из условий (1.6), соответствует решение $y:\left[t_{2}, \omega\left[\rightarrow \mathbb{R}\right.\right.$, где $t_{2} \in[a, \omega[$, дифференциального уравнения (1.1), которое допускает при $t \uparrow \omega$ асимптотические представления (3.28) и (3.29). Используя эти представления, условия (3.26), (3.27), нетрудно проверить, что каждое такое решение уравнения (1.1) является $P_{\omega}\left(Y_{0}, \ldots, Y_{n-1}, 1\right)$-решением.

Теорема 3.3 доказана.

\section{Литература}

1. Евтухов B. М., Самойленко А. М. Асимптотические представления решений неавтономных обыкновенных дифференциальных уравнений с правильно меняющимися нелинейностями // Дифференц. уравнения. 2011. - 47, № 5. - С. $628-650$.

2. Евтухов В. М., Клопот А. М. Асимптотика некоторых классов решений обыкновенных дифференциальных уравнений $n$-го порядка с правильно меняющимися нелинейностями // Укр. мат. журн. - 2013. - 65, № 3. C. $354-380$.

3. Евтухов В. М., Клопот А. М. Асимптотическое поведение решений обыкновенных дифференциальных уравнений $n$-го порядка с правильно меняющимися нелинейностями // Укр. мат. журн. -2011 . - 56, № 3 - $18 \mathrm{c}$.

4. Клопот А. М. Асимптотические представления решений дифференциальных уравнений $n$-го порядка с правильно меняющимися нелинейностями. - Дис. ... канд. физ-мат. наук. - 2014. - 148 с.

5. Сенета Е. Правильно меняющиеся функции. - М.: Наука, 1985. - 144 с.

6. Евтухов В. М., Самойленко А. М. Условия существования исчезающих в особой точке решений у вещественных неавтономных систем квазилинейных дифференциальных уравнений // Укр. мат. журн. - 2010. 62, № 1. - C. $52-80$.

Получено 26.10.2017 